\title{
How the Green's Functions May Be Used for Correcting Measurements of Transmission through Flat Homogeneous and Sandwich-Type Dielectric Sheets
}

\author{
Aleksey Karpov, Lubov Lesnaya, Ilya Milochkin, Sergey Shabunin ${ }^{\text {a) }}$ \\ Ural Federal University, Ekaterinburg, Russia (620000, Russia, Yekaterinburg, 19 Mira Street). \\ a) Corresponding author: shab@ieee.org
}

\begin{abstract}
The Green's functions of layered structures are used for analysis of measurement errors of transmitting losses through solid and sandwich-type dielectric structure. Influence of the spherical waves excited by radiators in the near zone is under investigation. As the simplest antenna, the Huygens element was used for calculations. The model of equivalent electric circuits was used for layered structure modelling. The transmitting losses of electromagnetic waves versus frequency for different dielectric sheets are shown. Influence of the distance between antennas on the transmitting losses measurements is analyzed. It is proposed to use the suggested method to analyze the accuracy of the transmission coefficients measurements if the horn antennas are used.
\end{abstract}

Keywords: measurements, propagation, transmitting losses, antenna radome, Green's function, diffraction.

\section{INTRODUCTION}

Solid monolithic and sandwich-type dielectric sheets are often used as the antenna radomes, frequency-selective surfaces, non-reflecting coves, and so on [1], [2]. Analytical methods and proper software such as the CST Microwave Studio, WIPL-D, HFSS, and FEKO based on numerical methods are applied to electromagnetic modelling the electromagnetic waves propagation through these structures [3] - [7]. In addition to the calculation methods, various methods of experimental determination of transmission and reflection coefficients are widely used in the antenna radome design [4], [5]. One of these methods is the method of measuring the transmission coefficient of the dielectric sheet by means of two antennas (Fig. 1). There are a few rules to measure the characteristics of the antenna shelters. When carrying out measurements, the condition of the far zone for the used antennas and material sample must be followed. The incident wave is needed to be flat near the dielectric slab. Sometimes, this condition is difficult to fulfill. In this paper, we consider the incidence of a spherical wave on the radome material sample under study and determine the magnitude of the measurement error in comparing with the flat wave.

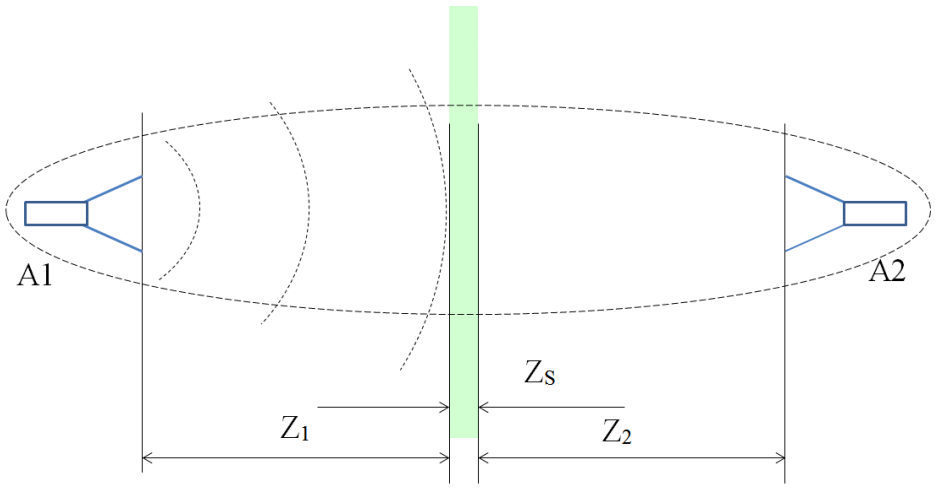

Fig. 1. Geometrical representation of the problem for the equivalent aperture method.

International Conference of Numerical Analysis and Applied Mathematics ICNAAM 2019

AIP Conf. Proc. 2293, 140014-1-140014-6; https://doi.org/10.1063/5.0026828

Published by AIP Publishing. 978-0-7354-4025-8/\$30.00 
The Green's function method is used for calculation of the electromagnetic wave propagation through solid and layered flat dielectric structures. Due to the accuracy and efficiency, the method is widely applied to solving the scattering and radiation problems [8], [9]. The suggested method may be applied both for the antenna radome design and for correcting measurements of the transmission and reflection coefficients. Electromagnetic reflection of the composite coatings can also be measured [10].

\section{ELECTRIC FIELD CALCULATION}

Application of the Green's functions to analysis of the plane layered structures is described in [11]. The idea of calculating the reflection and transmission is the following. The incident wave at the interface with the layered structure excites equivalent surface electric and magnetic currents. Radiation of these currents through the dielectric in the second medium is treated as the passage of electromagnetic waves. The transmission coefficient is calculated by comparing the amplitude of the incident and transmitting waves. Radiation of the equivalent currents in the first medium is treated as a reflection of the electromagnetic waves. The reflection coefficient is calculated. This model is suitable for infinitely far sources. As the Green's function takes into account the whole non-homogeneous structure under consideration, it is possible to calculate the transmission coefficient in the near zone, and then recalculate it in the case of a plane wave.

In this paper, the Huygens element is used as an electromagnetic source. The electrodynamic model of the Huygens element is a pair of orthogonal electric and magnetic dipoles (Fig. 2).

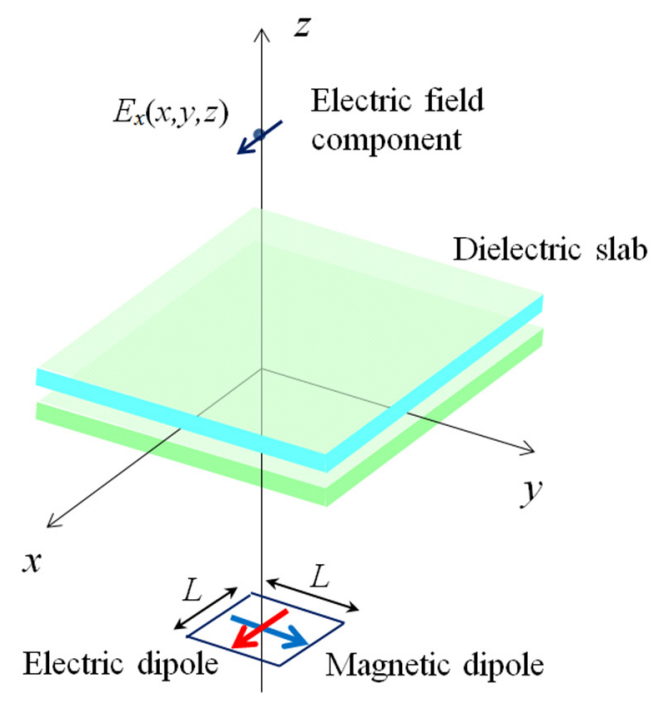

Fig. 2. The Huygens element with the electric (red) and magnetic (blue) dipoles radiates electromagnetic waves; the electric field component is calculated.

We believe that the electric dipole is oriented along the $x$-axis and magnetic dipole is oriented along the $y$-axis, and both are located at the point $z^{\prime}=-z_{1}$. The electrical field at the point of view $z=z_{s}+z_{2}$ is calculated as follows:

$$
\mathbf{E}(\mathbf{r})=\int_{S^{\prime}}\left[\overline{\bar{\Gamma}}_{11}\left(\mathbf{r}, \mathbf{r}^{\prime}\right) \mathbf{J}\left(\mathbf{r}^{\prime}\right)+\overline{\bar{\Gamma}}_{12}\left(\mathbf{r}, \mathbf{r}^{\prime}\right) \mathbf{M}\left(\mathbf{r}^{\prime}\right)\right] d s^{\prime}
$$

where for the Huygens element located at the source point $\left(x_{i}, y_{i}, z_{i}=-z_{1}\right)$, the electric dipole current is $\mathbf{J}\left(\mathbf{r}^{\prime}\right)=\vec{x}_{0} I_{0} \delta\left(y^{\prime}-y_{i}\right) \delta\left(z^{\prime}-z_{1}\right)$, the magnetic dipole current is $\mathbf{M}\left(\mathbf{r}^{\prime}\right)=\vec{y}_{0} I_{0} Z_{0} \delta\left(x^{\prime}-x_{i}\right) \delta\left(z^{\prime}-z_{1}\right) ; \quad \overline{\bar{\Gamma}}_{11}\left(\mathbf{r}, \mathbf{r}^{\prime}\right)$ and $\overline{\bar{\Gamma}}_{12}\left(\mathbf{r}, \mathbf{r}^{\prime}\right)$ are the proper Green's functions. Usually, the Huygens element is used for the aperture antennas modeling, such as the horn antennas. 
The transmitted field in the case under consideration has only $E_{x}$ component, so, only one component of the Green tensor for each dipole is used

$$
\begin{aligned}
& \Gamma_{11 ; x x^{\prime}}\left(\mathbf{r}, \mathbf{r}^{\prime}\right)=j \frac{\omega \mu_{0}}{4 \pi^{2}} \int_{-\infty}^{\infty} \int_{-\infty}^{\infty}\left[\frac{\xi^{2}}{k^{2}} \frac{\partial^{2} g\left(z, z^{\prime}\right)}{\partial z \partial z^{\prime}}+\eta^{2} f\left(z, z^{\prime}\right)\right] \frac{1}{\xi^{2}+\eta^{2}} \mathrm{e}^{-j \xi\left(x-x^{\prime}\right)} \mathrm{e}^{-j \eta\left(y-y^{\prime}\right)} d \xi d \eta, \\
& \Gamma_{12 ; x y^{\prime}}\left(\mathbf{r}, \mathbf{r}^{\prime}\right)=\frac{1}{4 \pi^{2}} \int_{-\infty}^{\infty} \int_{-\infty}^{\infty}\left[\eta^{2} \frac{\partial f\left(z, z^{\prime}\right)}{\partial z^{\prime}}-\xi^{2} \frac{\partial g\left(z, z^{\prime}\right)}{\partial z}\right] \frac{1}{\xi^{2}+\eta^{2}} \mathrm{e}^{-j \xi\left(x-x^{\prime}\right)} \mathrm{e}^{-j \eta\left(y-y^{\prime}\right)} d \xi d \eta,
\end{aligned}
$$

where the characteristic part of the Green's function associated with inhomogeneous medium along the $z$-axis is the following [9]:

$$
g\left(z, z^{\prime}\right)=\frac{\bar{I}_{1}\left(z^{\prime}, z_{1}\right) \vec{I}_{n+1}\left(z, z_{n}\right)}{j \omega \varepsilon_{0} \vec{Y}^{E}\left(z_{1}\right)} \prod_{i=1}^{n} T_{i}^{E}
$$

for the electric equivalent line associated with the electrical waves and

$$
f\left(z, z^{\prime}\right)=\frac{\bar{V}_{1}\left(z^{\prime}, z_{1}\right) \vec{V}_{n+1}\left(z, z_{n}\right)}{j \omega \mu_{0} \vec{Y}^{M}\left(z_{1}\right)} \prod_{i=1}^{n} T_{i}^{M}
$$

for the magnetic equivalent line associated with the magnetic waves. In (4) and (5), $\bar{I}_{1}, \vec{I}_{n+1}, \bar{V}_{1}$, and $\vec{V}_{n+1}$ are the modal currents and voltages in the equivalent electric and magnetic lines as in [9]; $\vec{Y}^{E}\left(z_{1}\right)=\vec{Y}^{E}\left(z_{1}\right)+\vec{Y}^{E}\left(z_{1}\right)$, $\vec{Y}^{M}\left(z_{1}\right)=\bar{Y}^{M}\left(z_{1}\right)+\vec{Y}^{M}\left(z_{1}\right)$ are the directional input admittances in the equivalent electric circuits, respectively. Since the source and the point of view are in different regions, the transmission coefficients $T_{i}^{E, M}$ of the $i$-th layer in the electric and magnetic equivalent circuits were added.

After performing the necessary transformations, we obtain for electric dipole the following expression:

$$
E_{x}\left(x=0, y=0, z=z_{s}+\mathrm{z}_{2}\right)=\frac{I_{0} L}{4 \pi^{2}} \int_{-\infty}^{\infty} \int_{-\infty}^{\infty}\left[\frac{\tilde{\xi}^{2}}{\vec{Y}^{E}\left(z_{1}\right)} \prod_{i=1}^{n} T_{i}^{E}+\frac{\tilde{\eta}^{2}}{\vec{Y}^{M}\left(z_{1}\right)} \prod_{i=1}^{n} T_{i}^{M}\right] \frac{k^{2}}{\tilde{\xi}^{2}+\tilde{\eta}^{2}} \mathrm{e}^{-j \gamma z_{1}} \mathrm{e}^{-j \gamma z_{2}} d \tilde{\xi} d \tilde{\eta},
$$

where $\gamma=k_{0} \sqrt{1-\tilde{\xi}^{2}-\tilde{\eta}^{2}}, L$ is the dipole length.

After transition of the complex plane from the Cartesian coordinate system to the cylindrical one, expression (6) is reduced to the following form:

$$
E_{x}=\frac{I_{0} Z_{0}}{\lambda} \frac{L}{\lambda} \int_{0}^{2 \pi} \int_{0}^{\infty}\left[\frac{\cos ^{2} \varphi}{\tilde{\tilde{Y}}^{E}\left(z_{1}\right)} \prod_{i=1}^{n} T_{i}^{E}+\frac{\sin ^{2} \varphi}{\tilde{\tilde{Y}}^{M}\left(z_{1}\right)} \prod_{i=1}^{n} T_{i}^{M}\right] \mathrm{e}^{-j \gamma z_{1}} \mathrm{e}^{-j \gamma z_{2}} \rho d \rho d \varphi
$$

where $\gamma=k_{0} \sqrt{1-\rho^{2}}$.

Expression (7) allows one to calculate the transmitting field passing through the layered dielectric structure with any number of layers.

For simplicity, let consider a homogeneous dielectric sheet with the thickness of $d_{1}$. The electric field component $E_{x}$ is defined as follows:

$$
E_{x}^{e}=\frac{I_{0} Z_{0}}{\lambda} \frac{L}{\lambda} \int_{0}^{2 \pi} \int_{0}^{\infty}\left[\frac{T_{1}^{E}}{\tilde{\tilde{Y}}^{E}\left(z_{1}\right)} \cos ^{2} \varphi+\frac{T_{1}^{M}}{\tilde{\tilde{Y}}^{M}\left(z_{1}\right)} \sin ^{2} \varphi\right] \mathrm{e}^{-j \tilde{\gamma} k_{0}\left(z_{1}+z_{2}\right)} \rho d \rho d \varphi
$$

for the electric field excited by the electric dipole and

$$
E_{x}^{m}=\frac{I_{0} Z_{0}}{\lambda} \frac{L}{\lambda} \int_{0}^{2 \pi} \int_{0}^{\infty}\left[\frac{T_{1}^{E}}{\tilde{\tilde{Y}}^{E}\left(z_{1}\right)} \cos ^{2} \varphi+\frac{\tilde{\gamma}^{2} T_{1}^{M}}{\tilde{\tilde{Y}}^{M}\left(z_{1}\right)} \sin ^{2} \varphi\right] \frac{1}{\tilde{\gamma}} \mathrm{e}^{-j \tilde{\gamma} k_{0}\left(z_{1}+z_{2}\right)} \rho d \rho d \varphi .
$$

for the electric field excited by the magnetic dipole.

The electrical field component $E_{i x}$ at the point of view $(x, y, z)$ excited by the Huygens element located at the source point $\left(x_{i}, y_{i}, z_{i}\right)$ is calculated using (7) and (8) as follows: 


$$
\begin{aligned}
E_{i x}=\frac{E_{0} L^{2} k_{0}^{2}}{4 \pi^{2}} \int_{0}^{2 \pi} \int_{0}^{\infty}(1+\tilde{\gamma})\left[\frac{1}{\tilde{\gamma}} \frac{T_{1}^{E} \cos ^{2} \varphi}{\tilde{\tilde{Y}}^{E}\left(z_{1}\right)}+\frac{T_{1}^{M} \sin ^{2} \varphi}{\tilde{\tilde{Y}}^{M}\left(z_{1}\right)}\right] \frac{\sin \left(0.5 k_{0} L \rho \cos \varphi\right)}{0.5 k_{0} L \rho \cos \varphi} \frac{\sin \left(0.5 k_{0} L \rho \sin \varphi\right)}{0.5 k_{0} L \rho \sin \varphi} \\
\times \mathrm{e}^{j k_{0} x_{i} \rho \cos \varphi} \mathrm{e}^{j k_{0} y_{i} \rho \sin \varphi} \mathrm{e}^{-j k_{0} x \rho \cos \varphi} \mathrm{e}^{-j k_{0} y \rho \sin \varphi} \mathrm{e}^{-j \tilde{\gamma} k_{0}\left(z_{1}+z_{2}\right)} \rho d \rho d \varphi
\end{aligned}
$$

where $L$ is the selected portion of the wave front, $E_{0}$ is the electric field strength at the point of the selected wave front.

For example, if the horn antenna is used for measurements as a radiator, we need take into account the amplitude and phase field distribution over the aperture. Thus, we have to sum the contribution of each of the elements of the aperture partition with coordinates $\left(x_{i}, y_{i}, z_{i}\right)$ at the observation point (10).

In (7) - (10), the normalized the input admittances in the equivalent electric and magnetic lines are

$$
\begin{gathered}
\tilde{\tilde{Y}}^{E}\left(z_{1}\right)=\frac{1}{\tilde{\gamma}}+\frac{\varepsilon_{1}^{\prime}}{\tilde{\gamma_{1}}} \frac{\tilde{\gamma_{1}} \cos \tilde{\gamma}_{1} k_{0} d_{1}+j \varepsilon_{1}^{\prime} \tilde{\gamma} \sin \tilde{\gamma}_{1} k_{0} d_{1}}{\varepsilon_{1}^{\prime} \cos \tilde{\gamma}_{1} k_{0} d_{1}+j \tilde{\gamma}_{1} \sin \tilde{\gamma}_{1} k_{0} d_{1}}, \\
\tilde{\tilde{Y}}^{M}\left(z_{1}\right)=\tilde{\gamma}+\tilde{\gamma}_{1} \frac{\tilde{\gamma} \cos \tilde{\gamma}_{1} k_{0} d_{1}+j \tilde{\gamma}_{1} \sin \tilde{\gamma}_{1} k_{0} d_{1}}{\tilde{\gamma}_{1} \cos \tilde{\gamma}_{1} k_{0} d_{1}+j \tilde{\gamma} \sin \tilde{\gamma}_{1} k_{0} d_{1}} .
\end{gathered}
$$

The transmission coefficients of one layer are

$$
\begin{gathered}
T_{1}^{E}=\frac{\varepsilon_{1}^{\prime} \tilde{\gamma}}{\varepsilon_{1}^{\prime} \tilde{\gamma} \cos \tilde{\gamma}_{1} k_{0} d_{1}+j \tilde{\gamma}_{1} \sin \tilde{\gamma}_{1} k_{0} d_{1}}, \\
T_{1}^{M}=\frac{\tilde{\gamma}_{1}}{\tilde{\gamma}_{1} \cos \tilde{\gamma}_{1} k_{0} d_{1}+j \tilde{\gamma} \sin \tilde{\gamma}_{1} k_{0} d_{1}},
\end{gathered}
$$

where

$$
\tilde{\gamma}=\sqrt{1-\rho^{2}}, \quad \tilde{\gamma}_{1}=\sqrt{\varepsilon_{1}^{\prime}-\rho^{2}} .
$$

For correct antenna measurements, it is important to define the far zone. The same kind of problems is needed to be taken into account for the frequency properties analysis of the sandwich-type or homogeneous dielectric sheets.

The transmitting losses of the dielectric sheet were calculated for different distance between the antennas and for various the dielectric constant of the sheet. The short distance was equal to $1 \mathrm{~m}$ (dash lines in Fig. 3). The long distance was equal to $40 \mathrm{~m}$ (solid lines in Fig. 3). The frequency was varied from $0.6 \mathrm{GHz}$ to $6 \mathrm{GHz}$.

The electric field amplitude of the transmitted waves through the dielectric sheet was compared with the case of the free space

$$
\text { Loss }=E(\text { with the sheet }) / E(\text { without the sheet }) .
$$

For the free space modelling the dielectric sheet with dielectric constant $\varepsilon_{1}^{\prime}=1$ was used. If there was no the dielectric sheet, the transmitting losses were equal to zero. Obviously, with increase in the permittivity of the sheet material, the oscillation of the dependences in Fig. 3 increases. At some frequencies, there is an effect of the signal amplification at the observation point at small distances between the antennas. On the charts, this is manifested as a shift in the values of the losses value into the negative region. At the same time, the value of losses in the areas of high reflection level decreases. This should be taken into account when measuring the transmission coefficient at small distances between the antennas of the order of several wavelengths in the case of the elementary radiators.

The transmission losses caused by the dielectric sheet are varied at small distance between antennas (Fig. 4). Small fluctuations are observed due to diffraction of the spherical incident wave from the small electric dipole at the distance about 2-3 wavelength. The transmission losses increase with the dielectric constant increasing. If the sheet thickness is about a half of the wavelength in the slab material, the transmission losses become less such as for $\varepsilon_{1}^{\prime}=18$ in Fig. 4 .

The suggested method allows calculating the field passing through the structure with an arbitrary number of layers, their thickness, dielectric and magnetic permeability. Losses in the dielectric material are also taken into account. 


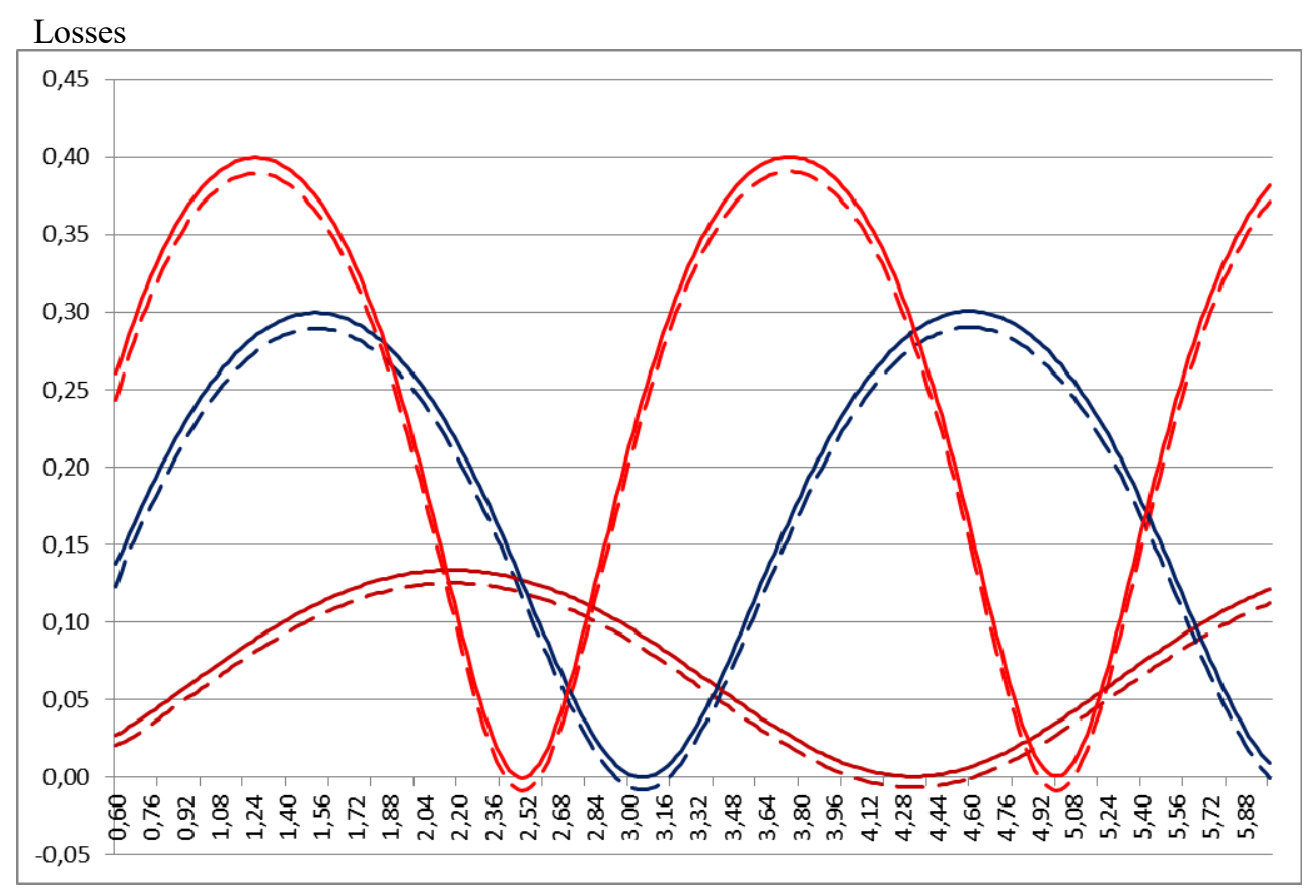

Frequency, $\mathrm{GHz}$

Fig. 3. The transmitting losses of electromagnetic waves versus frequency for different dielectric sheets with the equal thickness $\left(d_{1}=20 \mathrm{~mm}\right): \varepsilon_{1}^{\prime}=3$ (brown line), $\varepsilon_{1}^{\prime}=6$ (blue line), and $\varepsilon_{1}^{\prime}=9$ (red line); the distance between antennas is equal to $1 \mathrm{~m}$ (dash lines) and $40 \mathrm{~m}$ (solid lines).

It is not difficult to take into account the amplitude distribution of the incident field, that is, to take into account the radiation pattern of the antenna. The proposed approach makes it possible to take into account the polarization properties of the antennas involved in the measurements. The obtained analytical expressions for calculation of the field strength confirmed that the location of the measured sample between the antennas does not matter much. The total distance between the antennas affects. With its increase, the accuracy of the measurements increases.

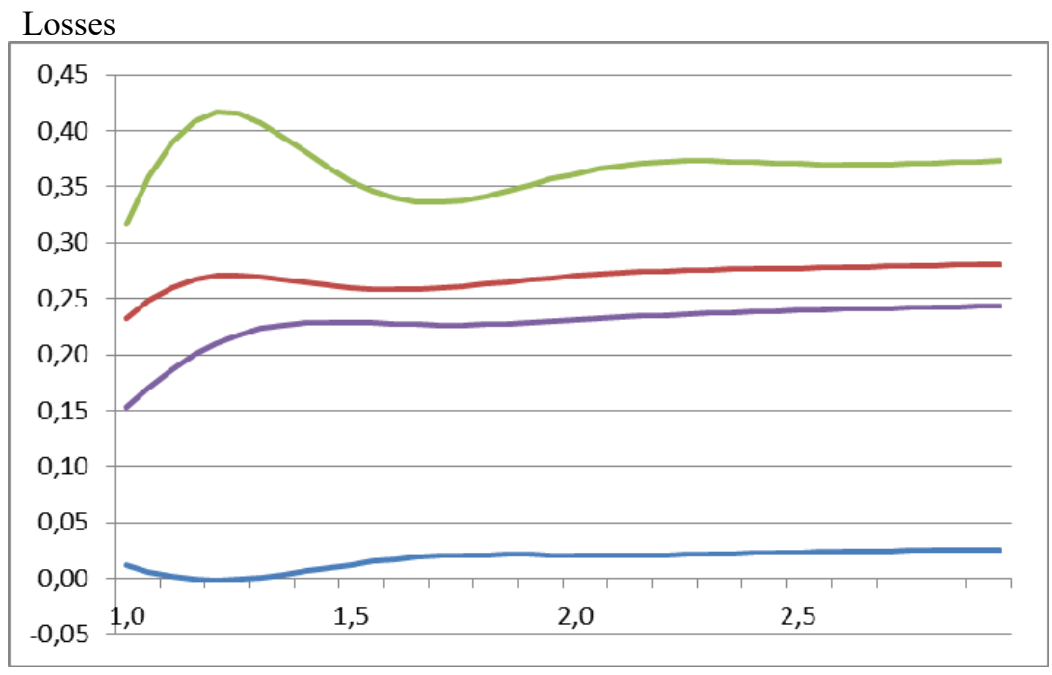

$\left(\mathrm{z}_{1}+\mathrm{z}_{2}\right) / \lambda$

Fig. 4. The transmitting losses of electromagnetic waves versus electrical distance between antennas ( $\left.d_{1} / \lambda=0.1\right): \varepsilon_{1}^{\prime}=2$ (blue line), $\varepsilon_{1}^{\prime}=6$ (brown line), $\varepsilon_{1}^{\prime}=12$ (green line), and $\varepsilon_{1}^{\prime}=18$ (purple line). 


\section{CONCLUSION}

The approach is proposed to estimating the accuracy of the planned measurements of the transmission coefficient through the plane homogeneous and layered dielectrics. The approach is based on application of the Green's functions of a stratified media. It is shown how the value of the field strength at the observation point changes due to the closing of the measuring antennas to the sample of the sheet dielectric. The sphericity of the wave front in the near zone distorts the measurement result. It is shown that location of the dielectric sheet between the antennas can be varied widely. The main affecting value is the distance between the antennas. Correct measurement of the characteristics of layered dielectrics increases the efficiency of their application as antenna shelters and increases the accuracy of design in specialized software.

\section{ACKNOWLEDGMENT}

This work was supported by the Grant of the Ministry of Science and Higher Education of the Russian Federation (project no 8.2538.2017/4.6).

\section{REFERENCES}

1. L.N. Ridenour, Radar Scanners and Radomes, (New York: McGraw-Hill Book Company, 1948).

2. D. J. Kozakoff, Analysis of Radome-Enclosed Antennas, (Norwood: Artech House, Inc.), 2010.

3. L. Sevgi, Electromagnetic Modeling and Simulation, (Wiley-IEEE Press, 2014).

4. J. Volakis, Antenna Engineering Handbook, (Fourth Edition, McGraw-Hill Education, 2007).

5. C.A. Balanis, Antenna Theory: Analysis and Design, (John Wiley \& Sons, Inc., 2016).

6. P. Mahima, M. Suprava, S. Vandana, P. S. M. Yazeen, U. N. Raveendranath, Electromagnetic Performance Analysis of Graded Dielectric Inhomogeneous Radomes, (Springer, 2018).

7. R. Shavit, Radome Electromagnetic. Theory and Design, (John Wiley \& Sons Ltd, 2018).

8. L. Vegni, R. Cicchetti, P. Capece, "Spectral dyadic Green's function formulation for planar integrated structures," IEEE Trans. Antennas Propagat., vol. 36, pp. 1057-1065, Aug. 1988

9. L. B. Felsen, N. Marcuvitz, Radiation and Scattering of Waves. (Englewood Cliffs: Prentice Hall, 1973).

10. M.G. Vakhitov, D.S. Klygach, "Reflection coefficient of the composites consisting of polyurethane matrix in 12-18 GHz", IEEE MTT-S International Microwave Workshop Series on Advanced Materials and Processes for RF and THz Applications, (IMWS-AMP 2017), 2018, January, pp. 1-3.

11. A. Karpov, S. Knyazev, L. Lesnaya, S. Shabunin, "Sandwich Spherical and Geodesic Antenna Radomes Analysis", Proc. of the 10th European Conf. on Antennas and Propag. (EuCAP 2016), 2016, Davos, Switzerland, pp. 1-5. 